BRAZZILLIAN JOURNAL

OF MEDICAL AND BIOLOGICAL RESHARCH

www.bjournal.com.br
ISSN 0100-879X

Volume 44 (11) 1070-1193 November 2011

BIOMEDICAL SCIENCES

AND

CLINICAL INVESTIGATION

Braz J Med Biol Res, November 2011, Volume 44(11) 1177-1183

doi: 10.1590/S0100-879X2011007500129

\title{
Antiretroviral therapy-associated dyslipidemia in patients from a reference center in Brazil
}

M.G.B. Ceccato, P.F. Bonolo, A.I. Souza Neto, F.S. Araújo and M.I.F. Freitas

The Brazilian Journal of Medical and Biological Research is partially financed by

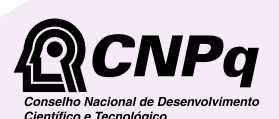

da Ciência e Tecnologia

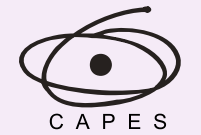

Ministério da Educação
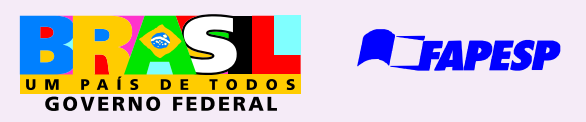

Institutional Sponsors
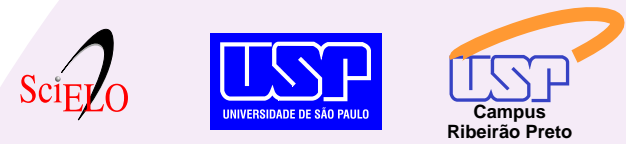

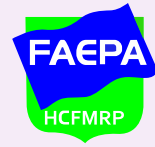

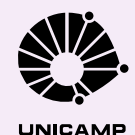

UNICAMP
Ф SHIMADZU

Explore High - Performance MS Orbitrap Technology In Proteomics \& Metabolomics

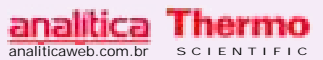




\title{
Antiretroviral therapy-associated dyslipidemia in patients from a reference center in Brazil
}

\author{
M.G.B. Ceccato ${ }^{1}$, P.F. Bonolo², A.I. Souza Neto ${ }^{3}$, \\ F.S. Araújo ${ }^{3}$ and M.I.F. Freitas ${ }^{4}$ \\ ${ }^{1}$ Departamento de Farmácia Social, Faculdade de Farmácia, Universidade Federal de Minas Gerais, \\ Belo Horizonte, MG, Brasil \\ 2Departamento de Ciências Médicas, Universidade Federal de Ouro Preto, Ouro Preto, MG, Brasil \\ 3Universidade José do Rosário Vellano, Belo Horizonte, MG, Brasil \\ ${ }^{4}$ Departamento de Enfermagem Materno-Infantil e Saúde Pública, Escola de Enfermagem, \\ Universidade Federal de Minas Gerais, Belo Horizonte, MG, Brasil
}

\begin{abstract}
The aim of this study was to determine the impact of antiretroviral therapy on the lipid profile of human immunodeficiency virus (HIV) patients before and after the initiation of highly active antiretroviral therapy (HAART). This was a cross-sectional analysis of patients receiving HAART at a reference center in Belo Horizonte, Brazil, on the basis of medical records from 2002 to 2006. Patients were included if they had at least one lipid test or a clinical or laboratory diagnosis of dyslipidemia/lipodystrophy. Among the 692 patients, 620 met the eligibility criteria. The majority were males (66.5\%), middle age (average 39 years), had a low educational level $(60.4 \%)$, and low income (51.0\%). HAART duration ranged from 11 days to 4.6 years, with a mean of 28.6 months (SD $= \pm 470.19$ days). The prevalence of dyslipidemia/lipodystrophy nearly tripled $(11.3 \%$ pre- and $32.4 \%$ post-HAART). Dyslipidemia was associated with older age $(\mathrm{P}=0.007)$, nucleoside reverse transcriptase inhibitor $(\mathrm{NRTI})+$ protease inhibitor $(\mathrm{PI})$ regimens $(P=0.04), N R T I+$ non-NRTI (NNRTI) regimens $(P=0.026)$, the use of stavudine $(d 4 T)$ in any regimen $(P=0.002)$ or in NRTI-based regimens $(P=0.006)$, and longer exposure to HAART $(P<0.000)$. In addition, there was no correlation between dyslipidemia and gender $(P=0.084)$. Only $2.0 \%$ of the patients received treatment for dyslipidemia during the trial. These results show a need for continuous monitoring of patients under antiretroviral therapy, particularly those using NRTI-based regimens, especially when combined with d4T and Pls. Secondly, interventions should be developed to correct metabolic changes.
\end{abstract}

Key words: Antiretroviral therapy; Dyslipidemia; Lipodystrophy; Management

\section{Introduction}

Metabolic changes such as the development of dyslipidemia, insulin resistance, glucose intolerance, and abnormal redistribution of body fat (loss of subcutaneous adipose tissue and increase of visceral fat), known as human immunodeficiency virus lipodystrophy syndrome (HIV-LS), represent a challenge in the treatment of HIV infection. These changes constitute risk factors associated with the development of cardiovascular diseases (1-3). Increased incidences of myocardial infarction (4), atherosclerotic disease and mortality related to cardiovascular events have been observed in people living with HIVIAIDS $(5,6)$. Cardiovascular diseases constitute the third or fourth leading cause of death among HIV-positive individuals in Europe (7). Moreover, lipodystrophic body changes can jeopardize the quality of life of patients, leading to low adherence to highly active antiretroviral therapy (HAART) and subsequent virologic and clinical failure $(8,9)$.

Despite the impact of the lipodystrophic syndrome on the treatment of HIV infection, and although this syndrome was identified in 1998 (1), little is known about its pathogenesis, prevention, diagnosis, and treatment. Furthermore, there is no precise definition of lipodystrophy, which leads to significant variation in reports on prevalence (20 to $80 \%$ ), incidence, risk factors, and response to interventions $(10,11)$. Current data suggest a multifactorial pathogenesis, with the major contributing factors being the choice of treatment and patient-related factors. The risk of developing this syndrome increases with the duration of treatment, patient's age, the level of immunodeficiency, changes in the viral load, and the type of antiretroviral regimen (12-14).

Correspondence: M.G.B. Ceccato, Departamento de Farmácia Social, FAFAR, UFMG, Av. Antonio Carlos, 6627, Sala 1048B2, 31270-010 Belo Horizonte, MG, Brasil. Fax: +55-31-3409-6849. E-mail: mgbceccato@farmacia.ufmg.br

Received September 18, 2010. Accepted September 23, 2011. Available online October 7, 2011. Published November $14,2011$. 
Dyslipidemia is a frequent side effect of antiretroviral therapy, especially in combinations that include protease inhibitors (PIs) and nucleoside reverse transcriptase inhibitors (NRTIs). Hypertriglyceridemia, already known to be associated with HIV infection (15), is the most common lipid abnormality; it occurs alone or in association with hypercholesterolemia, especially in patients with evidence of body fat abnormalities. Additionally, many HIV patients have low levels of high-density lipoprotein cholesterol (HDL-C), and these levels are further reduced by antiretroviral drugs.

Studies have shown that PIs and some NRTIs, especially stavudine (d4T), increase the levels of triglycerides (TG), total cholesterol (TC) and low-density lipoprotein cholesterol (LDL-C) but reduce HDL levels. In contrast, regimens with nevirapine and efavirenz (non-NRTIs - NNRTIs) show lower atherogenic effects on lipid profiles $(4,8,16)$. The effects of HAART on the pathogenesis of dyslipidemia are compounded by environmental, genetic, nutritional, and behavioral factors $(17,18)$.

The lack of a clearly defined pathogenesis for HIV-LS has limited the development of preventive and therapeutic strategies. Therefore, general recommendations include changes in diet and lifestyle, modification of antiretroviral therapy (replacing Pls with NRTIs or replacing d4T and zidovudine (AZT; NRTI), for example, with abacavir (ABC; NRTI) or tenofovir (TDF, NRTI) and the use of hypolipemics (19).

Patients on antiretroviral therapy should be monitored for cardiovascular events because, despite modifications in antiretroviral therapy, for example, replacing Pls with NRTIs and d4T and zidovudine (AZT; NRTI) with (ABC; $\mathrm{NRTI}$ ), and the resulting decrease in TG levels, they remain at increased risk $(5,18)$.

It is important to determine, recognize, follow-up, and treat metabolic changes and HIV-LS in individuals with HIVIAIDS. This has been well established, especially for patients with risk factors or with pre-existing lipid changes even before HAART, so as to prevent heart disease and other severe complications $(20,21)$.

Therefore, this problem poses a challenge to the reference centers that provide follow-up services to individuals living with HIV and AIDS, and it requires a multidisciplinary approach. The aim of the present study was to analyze the impact of antiretroviral therapy on the lipid profile of HIV patients following an antiretroviral regimen as established by the Brazilian Consensus (22), and to determine the prevalence of dyslipidemia/lipodystrophy pre- and postHAART.

\section{Material and Methods}

\section{Participants and design}

We conducted a cross-sectional analysis of patients receiving antiretroviral therapy at the Sagrada Família reference center, Belo Horizonte, MG. The study included patients with confirmed HIV infection who met Brazilian guidelines to initiate HAART (22), who were 18 years or older and who had received antiretroviral drugs at least once during the period of evaluation. Transfer from another reference center was the only exclusion criterion. The study was evaluated and approved by the Ethics Committee of Universidade Federal de Minas Gerais (322/06) and all patients gave written informed consent to participate.

\section{Exposure and outcome measurements}

Standardized and tested forms were used to collect data from medical charts. Patients were included if their records included results for at least one lipid test [TG, TC, HDL-C, LDL-C, and very low-density lipoprotein cholesterol (VLDL-C)] within a period of three months before or after the first HAART prescription (23). Additionally, patients who received a diagnosis of dyslipidemia/lipodystrophy after the first HAART prescription were included.

We recorded demographic characteristics (gender and age), clinical characteristics (time between the positive HIV test and the first antiretroviral (ARV) prescription, initial clinical classification [according to the definition of the Centers for Disease Control and Prevention, CDC, (24)], initial immunological profile (CD4+ lymphocyte count) and characteristics related to antiretroviral treatment (prescribed ARV regimen).

\section{Outcome variables}

Dyslipidemia was defined according to two criteria after the beginning of HAART: 1) laboratory data showing lipid levels above reference values according to the US National Cholesterol Education Program (NCEP III) (20) and IV Brazilian Guidelines (23), that is, TC $\geq 5.2 \mathrm{mM}$, HDL-C $<1.0 \mathrm{mM}$ (males), HDL-C $<1.3 \mathrm{mM}$ (females), $\mathrm{TG} \geq 1.7 \mathrm{mM}$, LDL-C $\geq 4.14 \mathrm{mM}$, and VLDL-C >1.0 mM, and 2) clinical criteria, such as having at least one recorded diagnosis of dyslipidemia/lipodystrophy.

Lipodystrophy was defined on the basis of changes in the redistribution of fat deposits and included both lipohypertrophy (visceral fat accumulation in the abdomen; accumulation of subcutaneous fat; accumulation of fat in the breasts; accumulation of fat in the cervical region (hump), and lipomas) and lipoatrophy (loss of fat in the legs, arms, gluteal region, and face; visible veins in upper and lower limbs). The diagnosis of lipodystrophy was established when the doctor identified and recorded at least one of these typical changes $(22,23)$.

\section{Statistical analysis}

A paired statistical test was used for the comparison of serum lipid levels pre- and post-HAART initiation. The Student $t$-test was used for independent samples and matched pairs. Nonparametric chi-square, Mann-Whitney, and Wilcoxon matched-pairs tests were used. For all tests, a $P$ value $<0.05$ was considered to be statistically signifi- 
cant. The variability in TC, HDL-C, LDL-C, VLDL-C, and TG levels was evaluated using the mean, standard deviation (SD) and interquartile variation (1st and 3rd quartiles). Epilnfo version 3.3.3 (CDC, USA) and SPSS ${ }^{\circledR}$ version 16 were used for data analysis, and Paradox DOS ${ }^{\circledR}$ version 4.5 was used for database maintenance.

\section{Results}

\section{Study population}

Among the 692 patients identified from the medical records, 620 met the eligibility criteria for the current analysis. No statistically significant difference was observed between participants and non-participants in terms of age, gender, education, income, or employment.

Of these 620 patients, the majority were males (66.5\%) and more than half were under 39 years of age $(55.2 \%$; median $=38$; mean $\pm \mathrm{SD}=39.2 \pm 9.9$ years $)$, ranging in age between 18 and 78 years. The majority lived alone $(68.8 \%)$ and had a low educational level $(60.4 \%$; median $=8.0$ years; mean $\pm \mathrm{SD}=7.6 \pm 3.9$ years $)$ and monthly income below US\$198 (51.0\%); $17.2 \%$ did not have a job when they started HAART.

\section{Dyslipidemia/lipodystrophy assessment}

The prevalence of dyslipidemia/lipodystrophy after initiation of HAART was $32.4 \%(95 \% \mathrm{Cl}=28.8-36.3 \%)$ compared to $11.3 \%$ before HAART $(95 \% \mathrm{Cl}=10.5-16.0 \%)$, a statistically significant difference (Table 1). Laboratory test results were available for 112 patients (18.1\%); 61 of them $(9.8 \%)$ showed alterations in lipid levels after HAART initiation, and $26.1 \%$ were diagnosed with dyslipidemia/lipodystrophy. In comparison, in the pre-HAART period, laboratory and clinical diagnoses of dyslipidemia/ lipodystrophy were found for $51(8.2 \%)$ and 24 patients (3.9\%), respectively; this difference was statistically significant (Table 1). For the comparisons of serum lipid levels pre- and post-HAART, a matched-pairs test showed that the means for all lipid values increased after HAART and that there was a statistically significant difference in total cholesterol and VLDL-C (Table 2).

The characteristics of the study population investigated according to the presence of dyslipidemia are shown in Table 3. Patients with dyslipidemia/lipodystrophy were generally older (average age 40.2 vs 38.5 years; $P=0.007$ ).

Based on clinical and immunological data (24), most participants had AIDS-related symptoms before the first ARV prescription (categories $B$ and $C=60.7 \%$ ). Among patients with dyslipidemia/lipodystrophy, most individuals had TCD4+ lymphocyte counts lower than 200 cells $/ \mathrm{mm}^{3}$ ( $P=0.010$; Table 3).

Forty-four different antiretroviral regimens were being prescribed at the center; they included NRTIs, NNRTIs, PIs, and fusion inhibitors. Nearly all regimens included an $\operatorname{NRTI}(99.7 \%)$, and stavudine was prescribed for $15.0 \%$ of patients. NNRTIs were included in $65.9 \%$ of the prescribed regimens. Most of the patients received a triple-drug regimen including two NRTIs plus an NNRTI (64.8\%) or a PI (31.8\%). The most frequent regimen consisted of AZT + lamivudine (3TC) with efavirenz [2 NRTIs + 1 NNRTI] (45.3\%). One hundred and ninety-nine patients $(32.1 \%)$ used a PI, with nelfinavir being the most frequently used $\mathrm{PI}(12.5 \%)$.

Table 3 shows the differences between ARV regimens and the association with dyslipidemia/lipodystrophy. It was noted that individuals using $\mathrm{Pl}$-based regimens with any

Table 1. Laboratory and clinical characteristics of the participants according to dyslipidemia diagnosis, Belo Horizonte, 2009.

\begin{tabular}{|c|c|c|c|c|}
\hline & \multicolumn{2}{|c|}{ Pre-HAART } & \multicolumn{2}{|c|}{ Post-HAART } \\
\hline & $\mathrm{N}$ & $\%$ & $\mathrm{~N}$ & $\%$ \\
\hline DyslipidemiaT & 70 & 11.3 & 201 & $32.4^{*}$ \\
\hline $\begin{array}{l}\text { Laboratory diagnosis of } \\
\text { dyslipidemia }\end{array}$ & 51 & 8.2 & 61 & $9.8^{*}$ \\
\hline Clinical diagnosis of dyslipidemia & 24 & 3.9 & 162 & $26.1^{*}$ \\
\hline Laboratory results $\mathbb{\pi}$ & 112 & 18.1 & 112 & 18.1 \\
\hline Total cholesterol\& & 11 & 9.8 & 19 & $17.0^{*}$ \\
\hline Triglycerides\& & 25 & 22.3 & 36 & $32.1^{*}$ \\
\hline HDL-C\& & 27 & 24.1 & 26 & 23.2 \\
\hline LDL-C\& & 2 & 1.8 & 3 & 2.7 \\
\hline VLDL-C $\&$ & 13 & 11.6 & 20 & $17.9^{*}$ \\
\hline
\end{tabular}

HAART = highly active antiretroviral therapy; HDL-C = highdensity lipoprotein cholesterol; LDL-C = low-density lipoprotein cholesterol; VLDL-C = very low-density lipoprotein cholesterol. IN refers to the total number of participants $(N=620) ;{ }^{\&} N$ refers to the lipid dosage records $(\mathrm{N}=112)$. ${ }^{*} \mathrm{P}<0.05$ compared to preHAART (chi-square test).

Table 2 - Pre- and post-HAART serum lipid levels $(N=620)$.

\begin{tabular}{lll}
\hline & \multicolumn{1}{c}{ Pre-HAART } & \multicolumn{1}{c}{ Post-HAART } \\
\hline${\text { Triglycerides }(\mathrm{mM})^{\mathrm{a}}}$ & $1.69(0.98-2.0)$ & $1.94(1.09-2.46)$ \\
${\text { Total cholesterol }(\mathrm{mM})^{\mathrm{a}}}$ & $4.19(3.20-4.96)$ & $4.69(4.02-5.31)^{\star}$ \\
HDL-C $(\mathrm{mM})^{\mathrm{b}}$ & $1.11(0.33)$ & $1.16(0.38)$ \\
LDL-C $(\mathrm{mM})^{\mathrm{b}}$ & $2.49(0.84)$ & $2.66(0.90)$ \\
VLDL-C $(\mathrm{mM})^{\mathrm{b}}$ & $0.78(0.56)$ & $4.46(3.47)^{\star}$ \\
\hline
\end{tabular}

Data for triglycerides and total cholesterol are reported as means (1st and 3rd quartiles) and for HDL-C, LDL-C and VLDL-C as means $( \pm S D)$. HAART = highly active antiretroviral therapy; HDL-C = high-density lipoprotein cholesterol; LDL-C = low-density lipoprotein cholesterol; VLDL-C = very low-density lipopro-

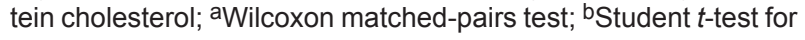
matched pairs; * $\mathrm{P}<0.05$ compared to pre-HAART. 
Table 3. Distribution of dyslipidemia in the patients studied.

\begin{tabular}{|c|c|c|c|}
\hline \multirow[t]{2}{*}{ Characteristics } & \multirow[t]{2}{*}{ Totala $^{\mathrm{a}}[\mathrm{N}(\%)]$} & \multicolumn{2}{|c|}{ Dyslipidemiab $[\mathrm{N}(\%)]$} \\
\hline & & Presence & Absence \\
\hline General population & 620 & $201(32.4)$ & \\
\hline \multicolumn{4}{|l|}{ Gender } \\
\hline Female & $208(33.5)$ & $64(30.8)$ & $144(69.2)$ \\
\hline Male & $412(66.5)$ & $137(33.3)$ & $275(66.7)$ \\
\hline Age (means $\pm S D)^{c}$ & $39.2 \pm 9.9$ & $40.2 \pm 10.4$ & $38.5 \pm 9.6^{*}$ \\
\hline Time between HIV test result and 1st ARV prescription (means $\pm S D)^{c}$ & $523.8 \pm 730.8$ & $539.4 \pm 757.8$ & $490.6 \pm 699.6$ \\
\hline \multicolumn{4}{|l|}{ Clinical classification } \\
\hline $\mathrm{B} / \mathrm{C}$ & $376(60.7)$ & $131(34.8)$ & $245(65.2)$ \\
\hline A & $243(39.3)$ & $70(28.8)$ & $173(71.2)$ \\
\hline \multicolumn{4}{|l|}{ TCD4+ lymphocyte count } \\
\hline$<200$ cells $/ \mathrm{mm}^{3}$ & $283(49.8)$ & $106(37.5)$ & $177(62.5)^{*}$ \\
\hline$\geq 200$ cells $/ \mathrm{mm}^{3}$ & $285(50.2)$ & $78(27.4)$ & $207(72.6)$ \\
\hline \multicolumn{4}{|l|}{ ARV with NRTI and NNRTI scheme } \\
\hline Yes & $402(64.8)$ & $118(29.4)$ & $284(70.6)^{*}$ \\
\hline No & $218(35.2)$ & $83(38.1)$ & $135(61.9)$ \\
\hline \multicolumn{4}{|l|}{ ARV with NRTI and PI scheme } \\
\hline Yes & $197(31.7)$ & $75(38.3)$ & $122(61.7)^{\star}$ \\
\hline No & $423(68.3)$ & $126(29.9)$ & $297(70.1)$ \\
\hline \multicolumn{4}{|l|}{ ARV with PI scheme } \\
\hline Yes & $199(32.1)$ & 75 (37.9) & $124(62.1)$ \\
\hline No & $421(67.9)$ & $126(30.0)$ & $295(70.0)$ \\
\hline \multicolumn{4}{|l|}{ Scheme with NNRTI } \\
\hline Yes & $408(65.9)$ & $121(29.7)$ & $287(70.3)^{*}$ \\
\hline No & $212(34.1)$ & $80(37.9)$ & $132(62.1)$ \\
\hline \multicolumn{4}{|l|}{ Scheme with D4T (NRTI) } \\
\hline Yes & $93(15.0)$ & $43(46.2)$ & $50(53.8)^{*}$ \\
\hline No & $527(85.0)$ & $158(30.0)$ & $369(70.0)$ \\
\hline \multicolumn{4}{|l|}{ Scheme with PI plus D4T (NRTI) } \\
\hline Yes & $43(6.9)$ & $22(51.2)$ & $21(48.8)^{*}$ \\
\hline No & $577(93.1)$ & $179(31.0)$ & $399(69.0)$ \\
\hline \multicolumn{4}{|l|}{ Scheme with D4T without IP } \\
\hline Yes & $48(7.7)$ & $18(37.5)$ & $30(62.5)$ \\
\hline No & $572(92.3)$ & $183(31.9)$ & $390(68.1)$ \\
\hline Duration of HAART use (days; means $\pm S D)^{c}$ & $857.3 \pm 470.2$ & $978.9 \pm 429.3$ & $799.1 \pm 478.2^{*}$ \\
\hline \multicolumn{4}{|l|}{ Duration of scheme with (days; means \pm SD) } \\
\hline $\mathrm{PIC}$ & $913.6 \pm 484.0$ & $1022.7 \pm 461.9$ & $847.1 \pm 486.9^{*}$ \\
\hline NNRTId & $830.0 \pm 460.0$ & $956.1 \pm 399.4$ & $777.3 \pm 473.6^{*}$ \\
\hline $\mathrm{D} 4 \mathrm{~T}^{\mathrm{C}}$ & $1034.0 \pm 445.4$ & $1121.5 \pm 384.5$ & $961.1 \pm 483.3$ \\
\hline D4T without $\mathrm{PI}^{\mathrm{C}}$ & $928.9 \pm 434.4$ & $1018.6 \pm 367.8$ & $875.1 \pm 467.6$ \\
\hline
\end{tabular}

HIV = human immunodeficiency virus; $\mathrm{ARV}=$ antiretroviral; NRTI = nucleoside reverse transcriptase inhibitors; NNRTI = non-

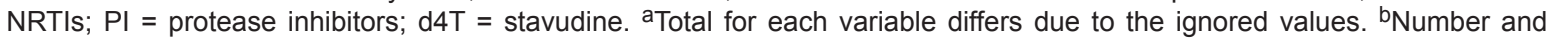
proportion of dyslipidemia in each variable category. Clinical classification according to Centers for Disease Control (24). Comparisons were made by the chi-square test except when otherwise indicated. ${ }^{\text {CANOVA test. }}{ }^{\mathrm{d}}$ Mann-Whitney test. ${ }^{*} \mathrm{P}<0.05$, presence of dyslipidemia compared to absence of dyslipidemia. 
other drug class presented a higher prevalence of dyslipidemia/lipodystrophy, especially when the regimen included d4T (51.2\%) versus any other drug class (31.0\%; $P=0.006)$. Alower prevalence of dyslipidemia/lipodystrophy was found among patients using NNRTI-based regimens (29.7\%) with any other drug class (37.9\%; $\mathrm{P}=0.041)$.

HAART duration ranged from 11 days to 4.6 years, with a mean of 28.6 months (SD $= \pm 470.19$ days) and a median of 30.1 months. Individuals who developed dyslipidemia/ lipodystrophy had a longer exposure to HAART compared to those who did not develop dyslipidemia/lipodystrophy (978.9 \pm 429.3 vs $799.1 \pm 478.2$ days; $\mathrm{P}<0.000$; Table 3 ).

\section{Discussion}

In this study, among the 620 individuals undergoing HAART, 201 (32.4\%) had at least one metabolic change and one diagnosis of dyslipidemia/lipodystrophy recorded in their medical charts. There was an approximately 3-fold increase in the prevalence of dyslipidemia/lipodystrophy in this population after the initiation of HAART compared to the pre-HAART period. Although the prevalence observed here is consistent with estimates reported by other investigators $(10,18)$, there is a great deal of variation in the literature, with estimates ranging from 18 to $83 \%(1,7-10,25)$.

There was a significant increase in the proportion of individuals with high levels of TG, TC and VLDL-C, while the proportion of individuals with high HDL-C remained the same after HAART. A matched-pairs statistical test comparing serum lipid levels pre- and post-antiretroviral treatment showed an increase in the mean values of all lipids and a decrease in the mean HDL-C level in patients receiving HAART, with no statistical significance for TG, HDL-C or LDL-C.

This finding is consistent with the results of Pujari et al. (8), who found that among patients with an average of 20 months of HAART, 41 and $45 \%$ developed hypertriglyceridemia and hypercholesterolemia, respectively. The authors pointed out that increased LDL-C is the principal risk factor for the development of coronary artery disease. Moreover, Bergersen et al. (7) found an increase in cholesterol and a decrease in HDL-C in patients using HAART when compared to a control group (no HAART). de Araújo et al. (26) pointed out an increase in TC and TG in patients receiving PIs. Carpentier et al. (27) reported a significant increase in VLDL-C after a short period of HAART (8 weeks). The DAD study (Data Collection on Adverse Events of Anti-HIV Drugs) found that hypercholesterolemia occurred in $27 \%$ of the patients using PIs, $23 \%$ of the patients using NRTIs and $10 \%$ of patients using NNRTIs, with the prevalence of hypertriglyceridemia being 40,32 , and $23 \%$, respectively, among these subgroups (5).

In the present study, after comparing serum lipid levels pre- and post-HAART initiation, a matched-pairs statistical test revealed an increase in mean TG values, with no statisti- cal significance. This finding may reflect, at least in part, the wide variability in the duration of antiretroviral therapy (11 days to 4.6 years) and the fact that a large proportion of patients used NNRTIs (65.9\%). Buchacz et al. (28) compared changes in lipid profiles over 24 months among adults and found that the mean TG levels were higher at 12 months compared to baseline levels $(P=0.40)$. Over 24 months of treatment, TG decreased by an average of $3.8 \mathrm{mg} / \mathrm{dL}$. The authors concluded that TC levels increased over the course of 24 months of NNRTI-based treatment but that relatively few patients developed high LDL-C or TG levels, although the overall HDL-C levels increased as well.

Our findings emphasize the need to establish strategies for the follow-up and control of dyslipidemia (29-31). Metabolic abnormalities can increase cardiovascular risk in some patients in the long term. Our results confirm the influence of other factors besides ARV toxicity on the development of dyslipidemia/lipodystrophy, such as age and severity of the infection, which have already been discussed in other studies $(10-13,25)$.

The metabolic changes resulting from HAART can also occur in patients who receive regimens containing NRTIs without Pls. This suggests that Pls may not be the only antiretroviral drugs implicated in the pathogenesis of these alterations $(13,17,25,26,28)$. Our findings corroborate this hypothesis. The patients exposed to PIs in regimens that also included any other drug class presented a higher prevalence of dyslipidemia/lipodystrophy $(P=0.051)$ compared to patients not exposed to PIs. The inclusion of reverse transcriptase analogues in PI-based regimens significantly increased the occurrence of dyslipidemia/lipodystrophy $(P$ $=0.041$ ) among the patients. Other studies have reported evidence that NRTIs are highly associated with dyslipidemia/ lipodystrophy $(9,13,25)$.

Some studies have suggested a synergism between the action of NRTIs and PIs $(8,24)$. Mallal et al. (11) showed that NRTIs contribute independently to the development of metabolic syndrome but also that PIs seem to be the major risk factors, probably acting in synergy with NRTIs. In our study, a strong association was observed between d4T exposure (in association with any ARV) and dyslipidemia/lipodystrophy when compared to patients without d4T exposure (46.2 vs $30.0 \%$; $\mathrm{P}=0.002$ ). The risk of dyslipidemia/lipodystrophy was increased when patients were exposed to the association of d4T with Pls in their regimen. Approximately $51.0 \%$ of these patients presented metabolic changes $(P=0.006)$. Among patients following $\mathrm{d} 4 \mathrm{~T}$ regimens without a $\mathrm{PI}$, the prevalence of dyslipidemia/ lipodystrophy dropped from 51.2 to $37.5 \%$. The results of this study suggest a synergistic effect of NRTIs and PIs, especially when d4T was included.

Antiretroviral regimens containing NNRTIs, especially nevirapine, have been associated with favorable changes in patients' lipid profiles (smaller increase in TC, TG and LDL-C and a proportionally greater increase in HDL-C) 
compared to PI-, NRTI- or efavirenz-based regimens $(3,25)$. The present results support these reports, with patients on an NNRTI-based regimen or an NNRTI + NRTI regimen presenting a lower proportion of dyslipidemia/lipodystrophy when compared with patients on other regimens (29.7 vs $37.9, \mathrm{P}=0.041$, and 29.4 vs $38.1 \%, \mathrm{P}=0.026$, respectively). However, controversy remains. Fontas et al. (32) found an association between NNRTI-based regimens and high levels of TC and LDL-C.

The longest duration of treatment observed was for d4T-based regimens (median 97.4 months). The duration of ARV use in our study population was sufficient to observe the development of ARV-related metabolic changes. Tomazic et al. (13) reported an association between longer duration of treatment and dyslipidemia. Our findings that the duration of antiretroviral treatment and the use of PI- and NRTI-based regimens significantly increased the prevalence of dyslipidemia/lipodystrophy corroborate the cited report (13). We should note that, even though patients on NNRTI-based regimens presented a lower prevalence of dyslipidemia/lipodystrophy, this prevalence increased with the duration of treatment $(P=0.001)$.

The prevalence of dyslipidemia/lipodystrophy increased 3-fold after the beginning of HAART. Patients receiving an NRTI + PI regimen or d4T (with any other ARV) had a higher rate of dyslipidemia/lipodystrophy than those treated with other regimens. The risk of dyslipidemia/lipodystrophy was exacerbated in patients previously exposed to a d4T $+\mathrm{PI}$ regimen. The mechanisms involved in $\mathrm{d} 4 \mathrm{~T}$ toxicity and the effects of its association with PIs deserve further investigation.

The limitations of the present study are primarily related to the data obtained from medical charts and laboratory tests. For example, TG analysis is not always conducted

\section{References}

1. Carr A, Samaras K, Burton S, Law M, Freund J, Chisholm DJ, et al. A syndrome of peripheral lipodystrophy, hyperlipidaemia and insulin resistance in patients receiving HIV protease inhibitors. AIDS 1998; 12: F51-F58.

2. Troll JG. Approach to dyslipidemia, lipodystrophy, and cardiovascular risk in patients with HIV infection. Curr Atheroscler Rep 2011; 13: 51-56.

3. Montessori V, Press N, Harris M, Akagi L, Montaner JS. Adverse effects of antiretroviral therapy for HIV infection. CMAJ 2004; 170: 229-238.

4. Fichtenbaum CJ. Coronary heart disease risk, dyslipidemia, and management in HIV-infected persons. HIV Clin Trials 2004; 5: 416-433.

5. Friis-Moller $\mathrm{N}$, Weber $\mathrm{R}$, Reiss $\mathrm{P}$, Thiebaut $\mathrm{R}$, Kirk $\mathrm{O}$, d'Arminio MA, et al. Cardiovascular disease risk factors in HIV patients - association with antiretroviral therapy. Results from the DAD study. AIDS 2003; 17: 1179-1193.

6. Villarroya F, Domingo P, Giralt M. Drug-induced lipotoxicity: lipodystrophy associated with HIV-1 infection and antiretrovi- in a uniform manner when it comes to making the patient observe a 12-h fast and to avoid alcohol for $72 \mathrm{~h}$ prior to the test. This could contribute to the large standard deviations that we observed for TG levels. However, public health services comply with the national standards for serum lipid measurements. Secondly, due to the three-month interval between HIV follow-up consultations, the cut-off period for retrospective data on lipid levels was three months, even though lipid changes were already established after eight weeks of ARV treatment (20).

Additionally, there was no information regarding the use of hypolipemic drugs, diabetes, hypothyroidism, diet, exercise, or other important lifestyle issues (smoking) during the study period.

The authors emphasize the need for continuous monitoring of patients on antiretroviral therapy, especially those on NRTI-based regimens and those using d4T and PIs. It should be pointed out that patients on NNRTI-based or NNRTI + $\mathrm{NRTI}$ regimens presented a lower risk of developing dyslipidemia/lipodystrophy. Interventions should be carried out to correct metabolic changes so as to attenuate the adverse effects of ARV on the health of HIV patients $(29,32)$.

\section{Acknowledgments}

This study is part of the "Early Intervention Project: strategies to increase adherence to antiretroviral therapy in patients followed at the HIV reference center", developed by the Department of Maternal-Child Nursing and Public Health of the Nursing School and the Department of Social Pharmacy of the School of Pharmacy, Federal University of Minas Gerais (UFMG), and the Department of Medical Sciences, Federal University of Ouro Preto (UFOP), MG, Brazil. Research supported by FAPEMIG. ral treatment. Biochim Biophys Acta 2010; 1801: 392-399.

7. Bergersen BM, Sandvik L, Bruun JN, Tonstad S. Elevated Framingham risk score in HIV-positive patients on highly active antiretroviral therapy: results from a Norwegian study of 721 subjects. Eur J Clin Microbiol Infect Dis 2004; 23: 625-630.

8. Pujari SN, Dravid A, Naik E, Bhagat S, Tash K, Nadler JP, et al. Lipodystrophy and dyslipidemia among patients taking first-line, World Health Organization-recommended highly active antiretroviral therapy regimens in Western India. $J$ Acquir Immune Defic Syndr 2005; 39: 199-202.

9. van Vonderen MG, Gras L, Wit F, Brinkman K, van der Ende ME, Hoepelman Al, et al. Baseline lipid levels rather than the presence of reported body shape changes determine the degree of improvement in lipid levels after switching to atazanavir. HIV Clin Trials 2009; 10: 168-180.

10. Carr A, Emery S, Law M, Puls R, Lundgren JD, Powderly WG. An objective case definition of lipodystrophy in HIVinfected adults: a case-control study. Lancet 2003; 361: 
726-735

11. Mallal SA, John M, Moore CB, James IR, McKinnon EJ. Contribution of nucleoside analogue reverse transcriptase inhibitors to subcutaneous fat wasting in patients with HIV infection. AIDS 2000; 14: 1309-1316.

12. Raimundo P, Miranda A, Ribeiro J, Mansinho K. [Cardiovascular risk in HIV infected patients]. Acta Med Port 2010; 23: 669-676.

13. Tomazic J, Silic A, Karner P, Vidmar L, Maticic M, Poljak M, et al. Lipodystrophy and metabolic abnormalities in Slovenian HIV-infected patients. Wien Klin Wochenschr 2004; 116: 755-759.

14. Jevtovic DJ, Dragovic G, Salemovic D, Ranin J, DjurkovicDjakovic $\mathrm{O}$. The metabolic syndrome, an epidemic among HIV-infected patients on HAART. Biomed Pharmacother 2009; 63: 337-342.

15. Grunfeld C, Pang M, Doerrler W, Shigenaga JK, Jensen P, Feingold KR. Lipids, lipoproteins, triglyceride clearance, and cytokines in human immunodeficiency virus infection and the acquired immunodeficiency syndrome. J Clin Endocrinol Metab 1992; 74: 1045-1052.

16. Kramer AS, Lazzarotto AR, Sprinz E, Manfroi WC. Metabolic abnormalities, antiretroviral therapy and cardiovascular disease in elderly patients with HIV. Arq Bras Cardiol 2009; 93: 561-568.

17. Friis-Moller N, Sabin CA, Weber R, d'Arminio MA, El-Sadr WM, Reiss $P$, et al. Combination antiretroviral therapy and the risk of myocardial infarction. N Engl J Med 2003; 349: 1993-2003.

18. Carr A. HIV lipodystrophy: risk factors, pathogenesis, diagnosis and management. AIDS 2003; 17 (Suppl 1): S141S148.

19. Tsiodras S, Poulia KA, Yannakoulia M, Chimienti SN, Wadhwa S, Karchmer AW, et al. Adherence to Mediterranean diet is favorably associated with metabolic parameters in HIV-positive patients with the highly active antiretroviral therapy-induced metabolic syndrome and lipodystrophy. Metabolism 2009; 58: 854-859.

20. Executive Summary of the Third Report of the National Cholesterol Education Program (NCEP) Expert Panel on Detection, Evaluation, and Treatment of High Blood Cholesterol in Adults (Adult Treatment Panel III). JAMA 2001; 285: 2486-2497.

21. Ronchini KR, Duarte AJ, Casseb JS, Gidlund M. Cardiovascular complications and increased levels of circulating modified low density lipoprotein in HIV patients and patients with lipodystrophy. Braz J Med Biol Res 2004; 37: 119-122.

22. Brasil. Ministério da Saúde. Coordenação Nacional de DST and AIDS. Consenso: recomendações para a antiretroviral therapy em adultos and adolescentes infectados pelo HIV, 2008. Available at [http://www.AIDS.gov.br]. Accessed November 18, 2009.

23. Sposito AC, Caramelli B, Fonseca FA, Bertolami MC, Afiune NA, Souza AD, et al. [IV Brazilian Guideline for Dyslipidemia and Atherosclerosis prevention: Department of Atherosclerosis of Brazilian Society of Cardiology]. Arq Bras Cardiol 2007; 88 (Suppl 1): 2-19.

24. Centers for Disease and Prevention. Revised classification system for HIV infection and expanded surveillance case definitions for AIDS among adolescents and adults. 1993. Available at [http://www.cdc.gov/mmwr/preview/mmwrhtml/ 00018871.htm]. Accessed September 22, 2006.

25. Carr A, Cooper DA. Adverse effects of antiretroviral therapy. Lancet 2000; 356: 1423-1430.

26. de Araújo PS, de Alencar X, Lopes CF, Duarte JY, da Silva MM, Carneiro EM. Antiretroviral treatment for HIV infection/ AIDS and the risk of developing hyperglycemia and hyperlipidemia. Rev Inst Med Trop São Paulo 2007; 49: 73-78.

27. Carpentier A, Patterson BW, Uffelman KD, Salit I, Lewis GF. Mechanism of highly active anti-retroviral therapy-induced hyperlipidemia in HIV-infected individuals. Atherosclerosis 2005; 178: 165-172.

28. Buchacz K, Weidle PJ, Moore D, Were W, Mermin J, Downing $R$, et al. Changes in lipid profile over 24 months among adults on first-line highly active antiretroviral therapy in the home-based AIDS care program in rural Uganda. J Acquir Immune Defic Syndr 2008; 47: 304-311.

29. Joly V, Flandre P, Meiffredy V, Leturque N, Harel M, Aboulker $\mathrm{JP}$, et al. Increased risk of lipoatrophy under stavudine in HIV-1-infected patients: results of a substudy from a comparative trial. AIDS 2002; 16: 2447-2454.

30. Pérez-Camacho I, Camacho A, Torre-Cisneros J, Rivero A. Factores de riesgo cardiovascular dependientes del tratamiento antirretroviral. Enferm Infecc Microbiol Clin 2009; 27 (Supl 1): 24-32.

31. Worm SW, Sabin CA, Reiss P, El-Sadr W, Monforte A, Pradier $\mathrm{C}$, et al. Presence of the metabolic syndrome is not a better predictor of cardiovascular disease than the sum of its components in HIV-infected individuals: data collection on adverse events of anti-HIV drugs (D:A:D) study. Diabetes Care 2009; 32: 474-480.

32. Fontas E, van Leth F, Sabin CA, Friis-Moller N, Rickenbach M, d'Arminio MA, et al. Lipid profiles in HIV-infected patients receiving combination antiretroviral therapy: are different antiretroviral drugs associated with different lipid profiles? J Infect Dis 2004; 189: 1056-1074. 